\title{
A Novel GPS/DR Integrated Navigation System for Electronic Road Pricing
}

\author{
Yu Zhenhua \\ Research \& Innovation Institute \\ Watchdata System Co.,Ltd \\ 7F, Qiming International Mansion, NO.101,Wangjing \\ Lize Middle Park, Chaoyang District, Beijing, China \\ 100102 \\ yu_zhen19427@sina.com
}

\author{
Yan Guangwen \\ Research \& Innovation Institute \\ Watchdata System Co.,Ltd \\ 7F, Qiming International Mansion, NO.101,Wangjing \\ Lize Middle Park, Chaoyang District, Beijing, China \\ 100102
}

\begin{abstract}
A novel vehicle navigation system is designed for electronic road pricing(ERP), which can charge by the distance of the route according to the traffic status. The method of elimination of outliers of the Global Positioning System(GPS) signal is presented. The combination of GPS and Dead Reckoning(DR) is fulfilled to solve the vehicle positioning in the blind area of the GPS, so that the integrated navigation system can provide real-time and continuous position in the city road. The system has been tested in the Singapore ERP2 project and achieved good test result.
\end{abstract}

\section{Keywords-GPS; DR; Information fusion; Time} Synchronization; ERP

\section{INTRODUCTION}

Traffic congestion toll is a economic measure of transport demand management, which plays a great role in relieving traffic jam in urban center areas and gains a special attention. As proven by worldwide practices, the measure of road congestion charging can be used to optimize the spatial and temporal distribution of traffic flow and improve the entire operation efficiency of road network. The key to reduce traffic congestion would be a comprehensive transport policy of which congestion pricing is a central component[1],[2]. .

Global Positioning System(GPS) provides a real-time, whole-day, and global navigation service[3],[4], which can be used to the city road congestion charging to locate the three-dimension position of the vehicle at the real time and charge by the mileage of the toll road section. As we know, the performance of GPS is influenced by surroundings in city roads. When the vehicle passes through the tall building, under the bridge, and tunnel etc. , the precision of position will be decreased greatly, even invalid.

Dead reckoning(DR) has many advantages such as independent navigation and high positioning accuracy in short time. But its disadvantage is that its error can be accumulated with the time and will be so great that it will go out of use.

As a consequence, Whatever positioning method we use, GPS or DR can't work well in urban road location separately[5]-[7]. We need to used both methods together to achieve a better position performance, in that we can not only use GPS to gain a stable and reasonable positioning property to make up for the accumulated errors of the DR, but also utilize the high short-time positioning precision of DR to compensate the error of GPS when the signal of GPS is blocked or interfered. Thus, the dynamic performance of positioning of the vehicle can be improved greatly and the high precision, reliability and stability are available. Consequently, we presents a novel GPS/DR integrated navigation system in order to provide a comprehensive positioning solution and good vehicle position performance in urban road.

\section{THE GPS/DR SYSTEM ARCHITECTURE}

The System realizes the functions of data acquisition and processing of GPS, the synchronization between GPS and DR, the DR algorithm and information fusion of GPS and DR. The architecture of system is shown as figure 1 .

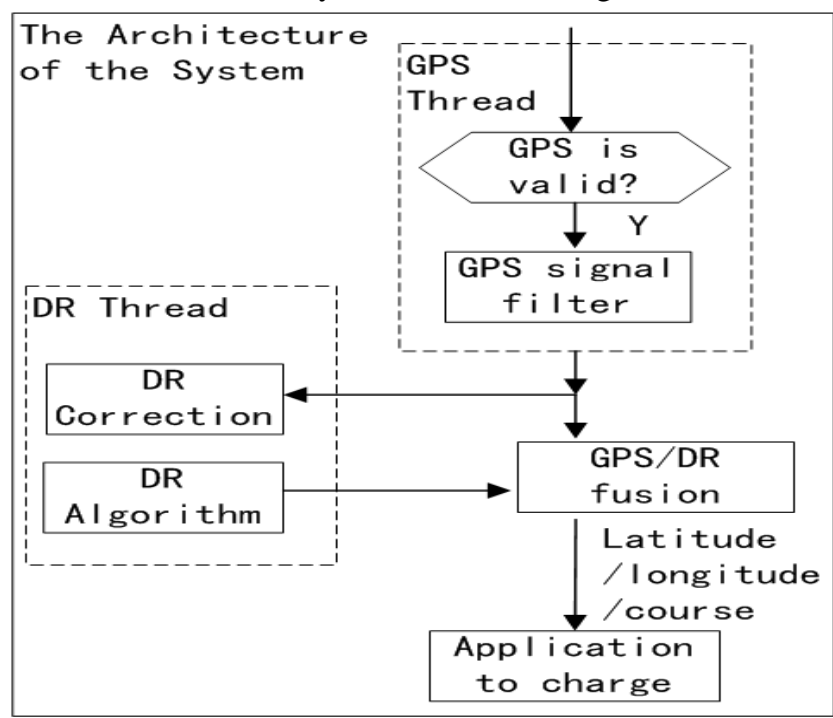

Figure 1. The architecture of GPS/DR navigation system.

The system use two thread to deal with the data of GPS and DR separately. When the DR keeps pace with the GPS, firstly we use GPS data, including latitude, longitude, speed and course, to verify DR parameter, secondly, we combine the positioning result of GPS and DR to raise the precision of location and plot the vehicle track on the electronic route map.

The data acquisition and processing of GPS consists of receiving GPS signal through serial communication from 
GPS receiver, parity check and extracting positioning data including latitude, longitude, speed and course.

The Inertial Measurement Unit(IMU) comprise tri-axial accelerometer and rate gyroscope, whose sampling rate are $100 \mathrm{~Hz}$. The accelerometer generates three dimension(3D) acceleration, and the gyroscope generates roll, pitch and course angular velocity. By use of these data, we can calculate the 3D attitude angle including course and complete the Dead Reckoning(DR) algorithm. By this way, the error of GPS is fixed when the signal of GPS is blocked or interfered.

The information fusion of GPS and DR uses cooperative fusion, namely, working out the weighted sum of positioning data of GPS and DR to acquire more accurate location value according to the positioning condition of the GPS.

\section{THE DEALING WITH OUTLIERS IN THE GPS}

The outliers refer to the wrong data due to sudden obstacle of the sensor or gross measurement errors[8]-[10].

The GPS receiver suffers the thermal noise, the circuit instability and the electromagnetic interference, thus, the outliers are generated, and need to be eliminated[11] The flowchart of elimination of GPS outliers is shown as figure 2.

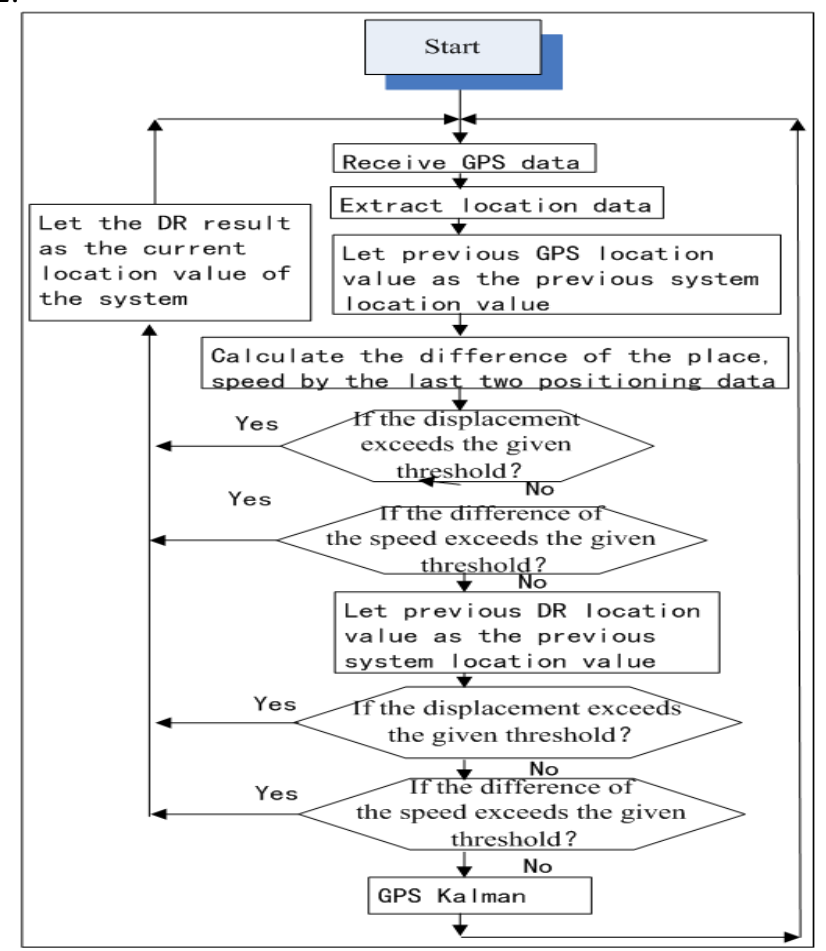

Figure 2. The elimiation of GPS outliers..

To kick of the outliers of GPS, we subtract the last location information, such as place and speed information, from the current one. If the difference exceeds a given threshold, we think of the location value as outliers, and use DR result to replace the current GPS data and make it as the location value of the system at the time.
To deal with the continuous outliers or the signal attack of GPS, we uses the last DR result as the last position value of the system , and compare it with the current GPS position value. If the difference of place, speed or acceleration is bigger than the given value, the current GPS location data is considered as continuous outliers. we can use DR result to replace the outliers, and take it as the current location value of the system.

\section{MATHEMATICAL MODEL OF GPS/DR}

Both state equation and observation equation of DR Kalman filter are given below.

\section{A. State equation of DR Kalman filter}

The carrier coordinate is adopted in DR system. we define geographical north-east-earth coordinate as vehicle carrier coordinate, where the origin is mass center of vehicle, $\mathrm{X}$-axis points to geographical north and $\mathrm{Y}$-axis points to the east.

The status variables- $\mathrm{X}$ of the DR filters is given below.

$$
X=\left[n, v_{n} a_{n}, e, v_{e} a_{e}\right]^{\prime},
$$

where, $n, v_{n} a_{n}$ refers to the place, speed and acceleration of the north, and $e, v_{e} a_{e}$ represents the place, speed and acceleration of the east.

Given that $\mathrm{T}$ is data sample rate of the system, we make a discretization equation and acquire the discrete status equation listed as the following.

$$
X_{k}=\Phi_{k, k-1} X_{k-1}+W_{k} \text { 。 }
$$

where , $X_{k}=\left[\begin{array}{lll}n_{k}, v_{n k} & a_{n k}, e_{k}, v_{e k} & a_{e k}\end{array}\right]^{\prime}$ is a discrete system status matrix, $\Phi_{k, k-1}$ is a discrete transfer matrix, and $W_{k}$ is a noise matrix. 。 The $\Phi_{k, k-1}$ is listed below.

$$
\Phi_{k, k-1}=\left[\begin{array}{cccccc}
1 & T & T^{2} / 2 & 0 & 0 & 0 \\
0 & 1 & T & 0 & 0 & 0 \\
0 & 0 & 1 & 0 & 0 & 0 \\
0 & 0 & 0 & 1 & T & T^{2} / 2 \\
0 & 0 & 0 & 0 & 1 & T \\
0 & 0 & 0 & 0 & 0 & 1
\end{array}\right]
$$

The discrete covariance equation matrix of the noise matrix $Q_{k}$ is listed below.

$$
Q_{k}=\left[\begin{array}{cccccc}
\delta_{n k}^{2} & 0 & 0 & 0 & 0 & 0 \\
0 & \delta_{v n k}^{2} & 0 & 0 & 0 & 0 \\
0 & 0 & \delta_{a n k}^{2} & 0 & 0 & 0 \\
0 & 0 & 0 & \delta_{e k}^{2} & 0 & 0 \\
0 & 0 & 0 & 0 & \delta_{v e k}^{2} & 0 \\
0 & 0 & 0 & 0 & & \delta_{a e k}^{2}
\end{array}\right]
$$


where, the elements in the diagonal line of the matrix means the variance of the noise.

\section{B. Observation equation of DR Kalman filter}

The measurement of the DR filter is

$$
Z=\left[w_{\mathrm{z}}, a_{n}, a_{e}\right]^{\prime} .
$$

where, $w_{\mathrm{z}}$ refers to the angular rate of the gyroscope、

$a_{n}$ is the north acceleration and $a_{e}$ is the east acceleration.

The observation equation is:

$$
Z(k)=H(k) X(k)+V(k)
$$

where,

$$
\begin{gathered}
H=\left[\begin{array}{cccccc}
0 & h_{12} & h_{13} & 0 & h_{15} & h_{16} \\
0 & 0 & 1 & 0 & 0 & 0 \\
0 & 0 & 0 & 0 & 0 & 1
\end{array}\right] \\
w_{z}=\frac{\partial}{\partial t}\left[\frac{v_{e}}{v_{n}}\right]+\xi_{w}=\frac{a_{e} v_{n}-v_{e} a_{n}}{\left(v_{e}^{2}+v_{n}^{2}\right)}+\xi_{w}
\end{gathered}
$$

$\xi_{w}$ is measurement noise.

Because the observation equation is nonlinear, we need to linearize it by Taylor series and neglect the higher-order terms, and we get the linear observation equation as below.

$$
\begin{aligned}
Z(k) \approx & H[\hat{X}(k, k-1)]+\frac{\partial H[\hat{X}(k, k-1)]}{\partial \hat{X}(k, k-1)}[X(k)-\hat{X}(k, k-1)] \\
& +V(k)
\end{aligned}
$$

where,

$$
\begin{gathered}
H=h_{k}[\hat{X}(k, k-1)]=\frac{\partial H[\hat{X}(k, k-1)]}{\partial \hat{X}(k, k-1)} \\
h_{12}=\frac{a_{e}\left(v_{e}^{2}+v_{n}^{2}\right)-\left(a_{e} v_{n}-v_{e} a_{n}\right) \cdot 2 \cdot v_{n}}{\left(v_{e}^{2}+v_{n}^{2}\right)^{2}} \\
h_{13}=\frac{-v_{e}}{\left(v_{e}^{2}+v_{n}^{2}\right)} \\
h_{15}=\frac{-a_{n}\left(v_{e}^{2}+v_{n}^{2}\right)-\left(a_{e} v_{n}-v_{e} a_{n}\right) \cdot 2 \cdot v_{e}}{\left(v_{e}^{2}+v_{n}^{2}\right)^{2}} \\
h_{16}=\frac{v_{n}}{\left(v_{e}^{2}+v_{n}^{2}\right)}
\end{gathered}
$$

\section{Information Fusion of GPS/DR}

GPS/DR integrated navigation system adopts a cooperative fusion, namely, working out the weighted sum of positioning data of GPS and DR to acquire more accurate location value according to the position condition of the GPS. The fusion diagram is shown as figure 3.

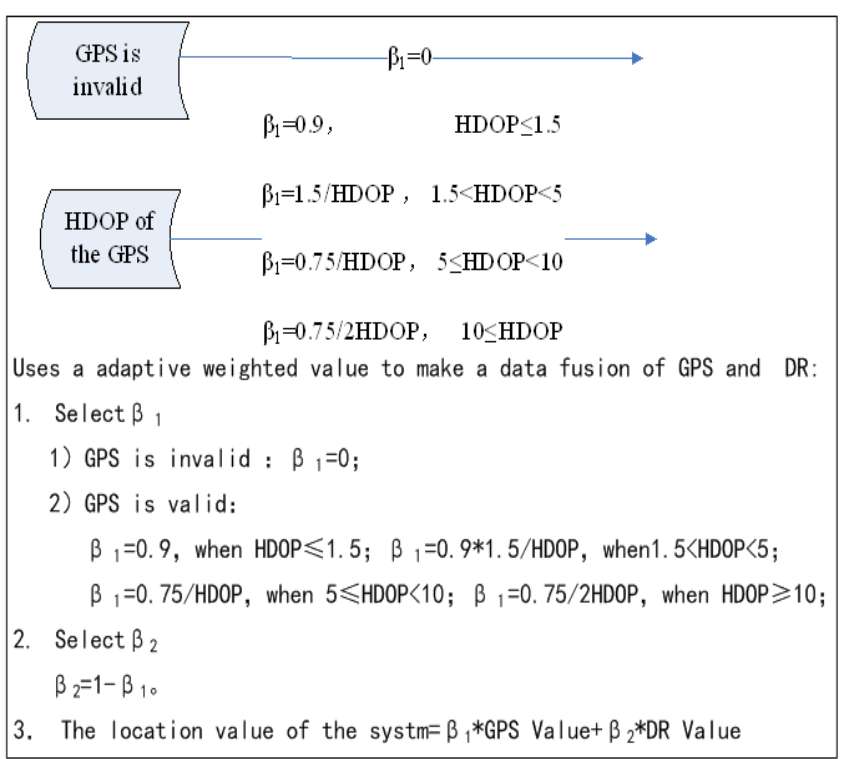

Figure 3. The data fusion of GPS and DR.

\section{SIGNAL SYNCHRONIZATION BETWEEN GPS AND DR}

It is important to make a synchronization between all sensors. In GPS/DR integrated navigation system, we need consider the signal synchronization between GPS and DR. Without the synchronization, these two navigation system exists time deviation and will make a data fusion at different time, thus, the error is produced[12],[13]. Therefore , the time synchronization is the basis to design the integrated navigation system.

Due to the importance of the time synchronization of the integrated navigation system, there are many researches made in this area[14]-[16].

The current time synchronization of the GPS/DR system is fulfilled by 1PPS of the GPS , which can achieve 1 millisecond time precision. But this method need to develop from the underlying implementation and is more complicated.

Therefore, we presents a relative simple method, which utilize the time information extracted from the NMEA0183 format or binary format navigation data output from GPS receiver. In order to improve time precision, we use binary format data to eliminate the time delay raised by the serial communication.

The NMEA0183 location data from GPS receiver include 'GPRMC' and 'GPGGA' message. By analyzing these two data, the whole position data, such as UTC(Universal Time Coordinated) time, latitude, longitude, height, course and speed, etc. can be extracted.

Since the total length of those two messages is less than 200 bytes, supposing the bit-rate of the serial communication is $115200 \mathrm{bps}$, the time delay is less than 14 milliseconds. If we use binary format, we can decrease the delay. For example, The UBLOX GPS receiver provide a binary format data named 'ubx' data. It uses 48 bytes to include the location data, so, the time delay of the serial communication is reduced to 3.3 milliseconds. 
Accordingly, we use binary format navigation message to extract the time information to realize synchronization. The flowchart of the time synchronization between GPS and DR is given by figure 4 .

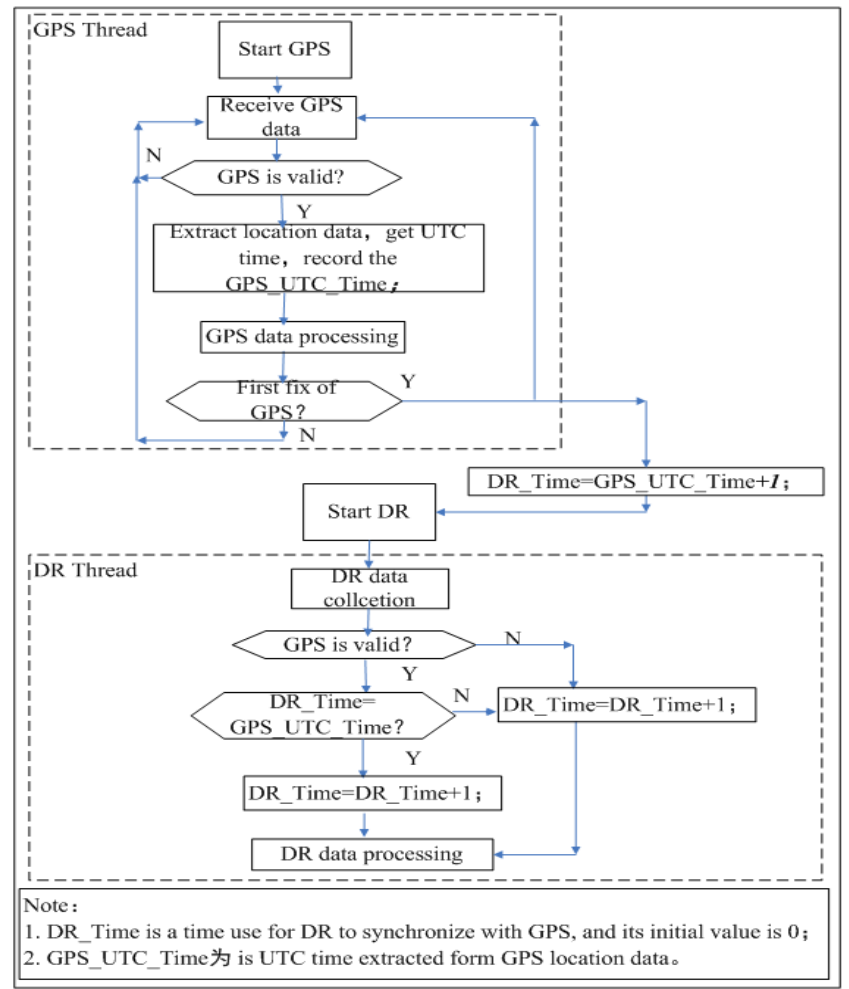

Figure 4. The time synchronization of GPS/DR navigation system.

\section{ROAD TEST OF GPS/ DR NAVIGATION SYSTEM}

In order to validate the positioning performance of the GPS/DR integrated navigation system, we held a road test at the capital airport road in Beijing. We directed our vehicle two circles around the road within 190 seconds.

To calibrate the position value, we adopted a high precision location device, named RTK(Real-time kinematic) device, whose positioning accuracy reaches 1 centimeter whatever the vehicle is still or moving. Thus, we can compare the location accuracy among the GPS, GPS/DR and RTK to evaluate the location performance of the GPS/DR navigation system.

The road tests include two cases. On one hand, DR runs independently and on the other hand, DR works with GPS to accomplish integrated navigation. After the tests, we compare these two positioning results with GPS and RTK.

The test result of the DR only is shown as figure 5 and figure 6.

The figure 5 gives the track of the vehicle and the figure 6 analyzes its error. From the figure 6, it is easy to see that when the DR works independently, it's positioning performance is good in short-time(within 25s or so), but its location error becomes worse with the time, and reaches 40 meters.

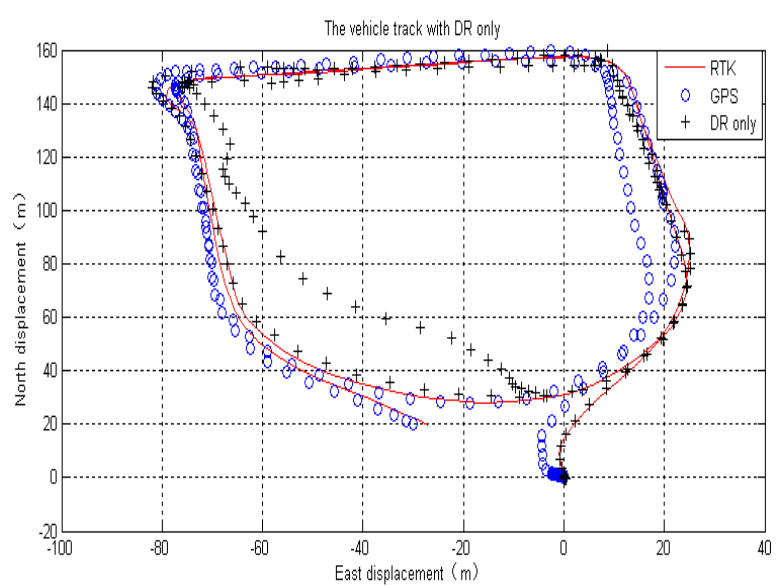

Figure 5. The comparison of track between GPS and DR only system.

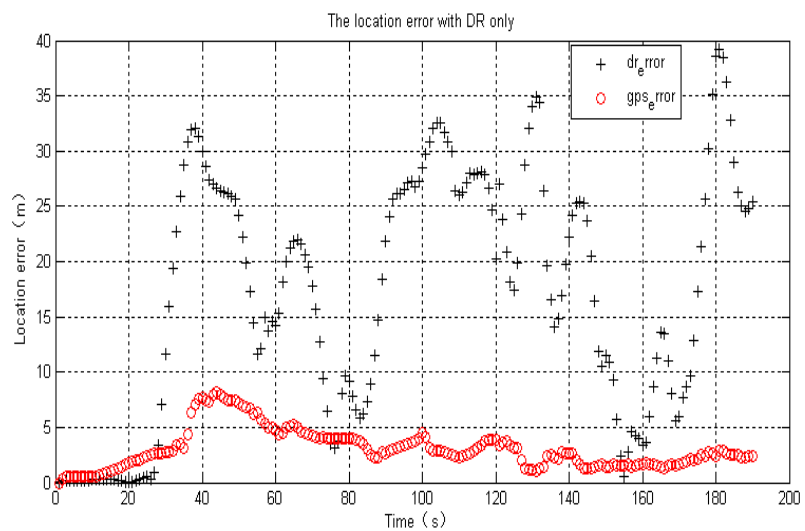

Figure 6. The comparison of the error between GPS and DR only

The test result of GPS/DR integrated navigation system is given by figure 7 and figure 8 . The figure 7 gives a vehicle track of the road test, and the figure 8 gives an analysis of error.

It is clear that the location accuracy is improved. The positioning error is decrease from $8 \mathrm{~m}$ of standalone GPS to $7 \mathrm{~m}$ of GPS/DR integrated system, and the entire positioning performance is made better.

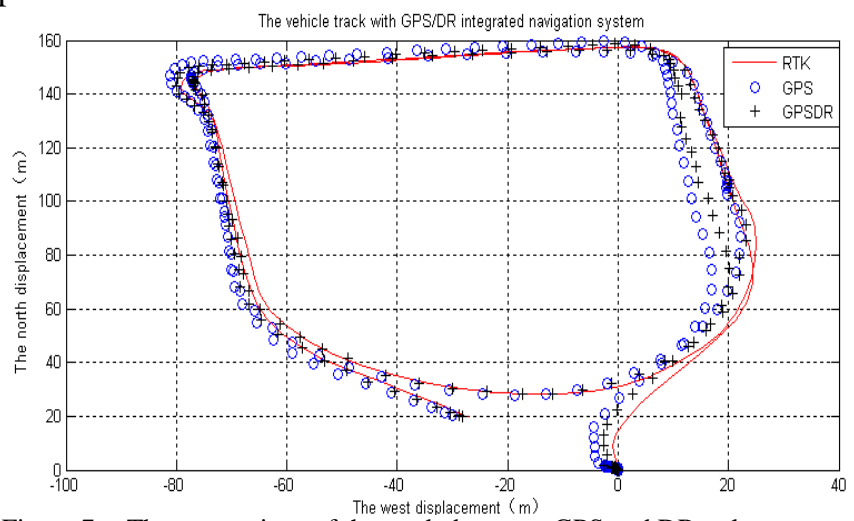

Figure 7. The comparison of the track between GPS and DR only system 


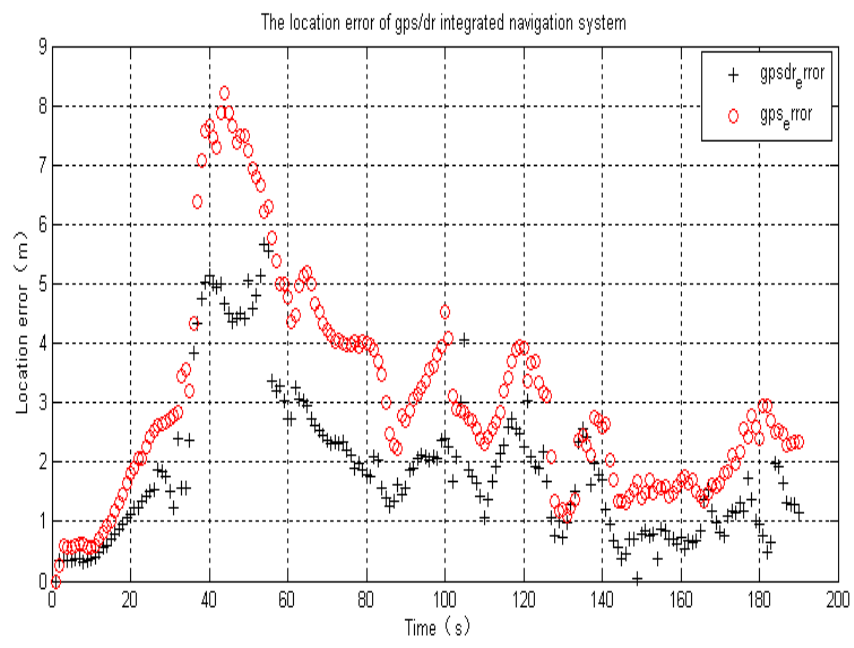

Figure 8. The comparison of the error between GPS and GPS/DR system.

Beside, we uses a DGPS(Differential GPS) in the system, thus, the accuracy is improved to $8 \mathrm{~m}$, whereas the accuracy of common GPS receiver is $15 \mathrm{~m}$.

The results of road tests show that the location performance of GPS/DR integrated navigation system is better than the standalone GPS system, and it can be used to urban road congestion pricing.

\section{CONCLUSION}

A GPS/DR integrated navigation system is designed which has high positioning precision and provides a solution to acquiring a good position performance when the GPS signal is blocked or invalid.

By use of GPS/DR integrated navigation system, the DR can work well and give a reasonable location value when the signal of GPS is blocked or interfered, even lost temporarily .

The GPS/DR integrated navigation system has been tested in Singapore ERP2 (The second generation of Electronic Road Pricing) project and achieved a good positioning test effect, so that the navigation system can be used to urban road charging to eliminate the traffic load and relieve city traffic jam.

\section{REFERENCES}

[1] Small K A, E T Verhoef, The Economics of Urban Transportation[M]. London and NewYork:Routledge.2007.

[2] Lindsey R.Do Economists Reach a Conclusion on Road Pricing? The Intellectual History of an Idea[J].Economic Journal Watch, 2006, 3(2): 292-379.

[3] Liu Jian-ye,Zeng Qin-hua, Zhao Wei, et al. The theory and application of Navigation System[M]. XI'AN:Northwestern Polytechnical University Press, 2010.

[4] Tan Shu-Seng.The engineer of Satellite Navigation[M] Beijing:National Defence Industry Press,2010

[5] Guan Guixia, Qiu Dehui, Lan Xiaoting. Information fusion on GPS/DR Integrated navigation[J]. Computer Applications and Software, 2006, 23(5): 54,55,123.

[6] WANG Lei, ZHANGHuaizhou . Indirect Kalman Filter ApplicationonCNS / SINSIntergatedNavigationSystem [J]. Measure and Contorl Technique, 2008， 27(3) : 88-90

[7] Liu Jun, Shi Yun-bo, Li jie. Micro-Inertial technique[M]. Beijing:Publishing House of Electronics Industry, 2005

[8] WU Hui-ying WU Lian-da. An improvement method for data preprocess[J]. ACTA ASTRONOMICA SINICA,2005,46(4): 433-440.

[9] ZHU Zhuan-min, QIU Hong-xing, LI Ji-sheng, et al. Identification and elimination of outliers in dynamic measurement data[J].Systems Engineering and Electronics, 2004,26(2): 147-149

[10] YAN Xin, LIU Jie-yu, NING Xiao-lei, et al. Application of Adaptive Kalman Filter Tolerant to Outliers in MEMS Accelerom eter Signal Processing[J]. Electronics Optics\& Control, 2009,16(11): 71-73.

[11] XU Li-jia ,CHEN Yang-zhou ,CUI Ping-yuan. Study on Information Fusion Technology in GPS/INS Integrated Navigation System[J]. Computer Simulation, 2004,21(5): 20-24

[12] SKOG I. Handel P. Effects of time synchronization errors in GNSS-aided INS[C] //Position. Location and Navigation Symposium, 2008 IEEE/ION.

[13] DING Wei-dong, WANG Jin-ling, LI Yong,et al. Time synchronization error and calibration in integrated GPS/INS systems[J]. ETRl Journal.2008, 30(1):59-66

[14] LEE H K.LEE J G. JEE G. Calibration of measurement delay in global positioning systen/ strapdown inertial navigation system[J]. Journal of Guidance. Control and Dynamics, 2002, 25(2): 240-247

[15] LI Qian, ZHAN Xing-qun, WANG Li-duan. Time Synchronizer Design in Integrated GPS/INS System[J] . CHINESE JOURNAL OF SENSORS AND ACTUATORS, 2009, 22(12): 1752-1756.

[16] ZHU Zhi-qin, WU Yu-hong, YANG Yuan-xin. Modulari Practice of Time Synchronization in Integrated GPS/INS Systems[J]. Geomatics and Information Science of Wuhan University,2010, 35(7);830-832. 